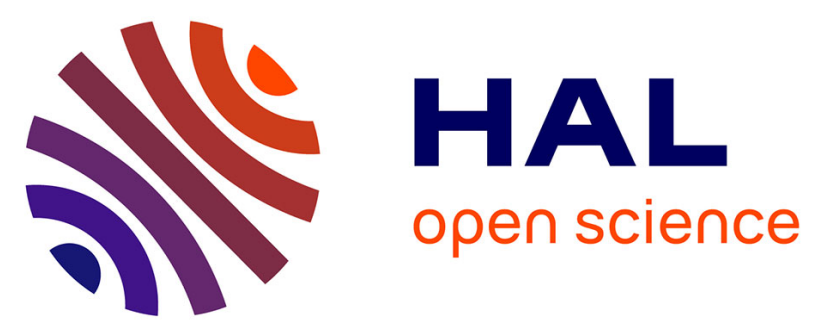

\title{
Synergy of Mn and Co in slab-based nanocomposites for hybrid supercapacitors: impact of restacking process on electrochemical properties
}

Celine Tang, Domitille Giaume, François Weill, Nicolas Penin, Marie Anne

Dourges, Hassan Saadaoui, Liliane Guerlou-Demourgues

\section{To cite this version:}

Celine Tang, Domitille Giaume, François Weill, Nicolas Penin, Marie Anne Dourges, et al.. Synergy of $\mathrm{Mn}$ and Co in slab-based nanocomposites for hybrid supercapacitors: impact of restacking process on electrochemical properties. ACS Applied Energy Materials, 2019, 2 (11), pp.7832-7842. 10.1021/acsaem.9b01263 . hal-02382879

\section{HAL Id: hal-02382879 \\ https://hal.science/hal-02382879}

Submitted on 1 Oct 2020

HAL is a multi-disciplinary open access archive for the deposit and dissemination of scientific research documents, whether they are published or not. The documents may come from teaching and research institutions in France or abroad, or from public or private research centers.
L'archive ouverte pluridisciplinaire HAL, est destinée au dépôt et à la diffusion de documents scientifiques de niveau recherche, publiés ou non, émanant des établissements d'enseignement et de recherche français ou étrangers, des laboratoires publics ou privés. 


\title{
Synergy of $\mathrm{Mn}$ and Co in slab-based nanocomposites for hybrid supercapacitors: impact of restacking process on electrochemical properties
}

\author{
Tang C. ${ }^{1,3}$, Giaume D. ${ }^{2,3}$, Weill F. ${ }^{1}$, Penin N. ${ }^{1}$, Dourges M.-A. ${ }^{4}$, Saadoui H. ${ }^{5}$, Guerlou-Demourgues L. ${ }^{1,3}$ \\ ${ }^{1}$ CNRS, Univ. Bordeaux, ICMCB, UMR 5026, F-33600 Pessac, France \\ ${ }^{2}$ Chimie-ParisTech, PSL Research University, CNRS Institut de Recherche de Chimie-Paris (IRCP), 75005 Paris, France \\ ${ }^{3}$ RS2E, Réseau Français sur le Stockage Électrochimique de l'Énergie, FR CNRS 3459 \\ ${ }^{4}$ Institut des Sciences Moléculaires, Université de Bordeaux, UMR 5255, CNRS, 33405 Talence, France \\ ${ }^{5}$ CRPP-CNRS, Université de Bordeaux, UMR 5031, F-33600 Pessac, France
}

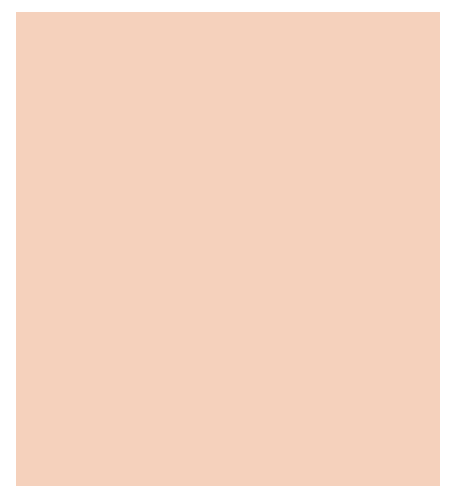

\begin{abstract}
To develop materials with enhanced electrochemical properties, an original synthesis strategy based on the exfoliation and restacking of manganese and cobalt layered transition metal oxides has been explored. Successful exfoliation yielded "building nanoblocks", which were further reassembled in mixed nanocomposites, by modifications of electrostatic interactions in solution. Three methods were tested, differing by the acidification procedure and time of mixing. The restacking conditions play a key role on the microstructural homogeneity of the nanocomposites. Such a nanocomposite approach marrying a good electronic conductor (cobalt oxyhydroxide) with a pseudocapacitive material (manganese oxide) should thus be very beneficial for development of highly efficient pseudocapacitors.
\end{abstract}




\section{Introduction}

The ever-increasing demand of renewable energies imposes, due to their intermittent nature, the development of performant energy storage devices to sustain it.(1) Among the various solutions, energy can be stored electrochemically in batteries and supercapacitors. Although batteries currently present higher energy density, their relatively lower power density and lower cycle life limit their use in high power applications.(2-4) In contrast, supercapacitors enable access to much higher power density.(5) They are particularly adapted for applications requiring energy pulses in short periods of time. Most supercapacitors commercially available today are symmetric EDLC (electric double layer capacitance) devices $(6,7)$ that rely on an electrostatic charge storage mechanism. The electrodes are based on materials presenting a high specific surface area, typically carbon-based materials such as activated carbon $(\mathrm{AC}),(8)$ graphene, $(9,10)$ carbon nanotubes $(\mathrm{CNT}),(11)$ or their composites.(12) The main advantage these materials offer is related to their low cost and high electrical conductivity, inducing high power applications.(7) However, they still require improvements, especially regarding their energy density. An alternative to EDLC electrodes is to use pseudocapacitive electrodes, where the charge storage is caused by redox reaction at the electrode surface in a faradaic process.(13) This phenomenon was first been observed by Conway on $\mathrm{RuO}_{2}$ materials, $(13,14)$ and shortly after on other transition metal oxides, $(15,16)$ such as $\mathrm{MnO}_{2},(17-19)$ or intrinsically conductive polymers, $(20,21)$ such as polythiophenes (PEDOT),(22,23) or polypyrrole (Ppy).(24,25) The charge and discharge processes involve redox reactions between various valence states of specific elements in these oxides. Thanks to this faradaic charge storage mechanism, pseudocapacitors possess relatively higher energy density than EDLCs but a significantly lower power density. This is especially true for $\mathrm{MnO}_{2}$-based electrodes,(19,26-30) which have attracted a lot of attention these last decades, mainly due to their excellent pseudocapacitive behavior at moderate regime. However, their electronic conductivity is very low,(19) thus increasing the charge transfer resistance which in turn decreases the power density and rate capability.

In recent years, some intensive studies have been dedicated to enhancement of the electrochemical performances of $\mathrm{MnO}_{2}$ electrodes. Two main strategies can be considered: boosting the specific surface area $(26,31)$ or enhancing the electronic conductivity.(32-34) In the first case, increasing the surface area means higher surface activity and thus more electroactive sites, leading to more efficient use of electrode materials. The most promising methods for achieving such structures is by a self-assembly process that can be template-assisted. $(35,36)$ For example, a hard template of $\mathrm{SiO}_{2}$ spheres can be used as a scaffold for the deposition of metal oxide $\mathrm{MnO}_{2}$ to define the shape of the desired nanomaterial.(35) Followed by hydrothermal treatment (in a $\mathrm{NaOH}$ solution), hollow $\mathrm{MnO}_{2}$ nanospheres can be obtained. The as-prepared hollow $\mathrm{MnO}_{2}$ nanospheres exhibit better capacitive behavior and cycling stability than their micronic $\mathrm{MnO}_{2}$ homologue.

Another very effective way of boosting the specific surface area is to perform exfoliation. This strategy is limited to $2 \mathrm{D}$ layered materials (for example, graphene $(37,38)$ ) and can be achieved by multiple liquid techniques based on ion exchange, $(39,40)$ ion intercalation,(41) or sonication-assisted exfoliation.(42) Thus, the study of swelling and exfoliation of layered manganese oxide has attracted a lot of attention,(43-45) as the obtained materials present high specific surface area, with enhanced potential for pseudocapacitor applications.

The second strategy to achieve better electrochemical performances of $\mathrm{MnO}_{2}$ at high regime is to enhance its electronic conductivity. To do so, forming hybrid $\mathrm{MnO}_{2}$-based structures with highly conductive carbons is one of the most adopted methods as they show good electrical conductivity as well as excellent mechanical and electrochemical stability. This has been conducted with carbon nanotubes/nanofibers/spheres, $(46,47)$ as well as graphene.(48,49) Conducting polymers, such as PEDOT, PANI, or PPy,(50-53) are also promising materials to be coupled with $\mathrm{MnO}_{2}$, as they offer good conductivity, controllable and easy synthesis, and uniform and porous structures that lead to good pseudocapacitive properties. Another effective way to improve electrical conductivity and to boost electrochemical performances is to synthesize $\mathrm{MnO}_{2}$-conductive metal composites, such as composites with noble metals $(54,55)$ or other transition metals.(56-58) In this frame we aim at designing nanocomposites mixing birnessite manganese oxide, known for its pseudocapacitive properties, and cobalt oxyhydroxide containing $\mathrm{Co}^{4+}$, exhibiting good electronic conductivity, in order to get a synergy effect and to improve the electrochemical properties.

We offer a synthesis strategy that aims at enhancing both the specific surface area (by nanostructuration through exfoliation) and the electronic conductivity of $\mathrm{MnO}_{2}$. For that purpose, we propose to build a composite at the nanometer scale by combining birnessite $\mathrm{MnO}_{2}$ phases, which are known to exhibit the best specific capacitance among the different $\mathrm{MnO}_{2}$ varieties(29) and $\beta$ (III) type layered cobalt oxyhydroxides (denoted as $\beta 3-\mathrm{CoOOH}$ ), which are expected to act as conductivity enhancers for birnessite, as suggested by the extensive studies on their enhanced electronic conductivity and interesting faradaic properties. $(59,60)$ Based on 2D materials, this strategy consists of (i) the synthesis of the starting layered materials chosen for their complementary properties, (ii) the exfoliation of these layered objects in order to obtain "building nanoblocks" followed by (iii) their fine restacking, 
inducing nanostructuration. This "nano-architectural" approach of material synthesis preserves the layered structure of materials, whereby a synergistic effect can be achieved. The desired combination of excellent pseudocapacitive properties of manganese oxide as well as high electronic conductivity of cobalt oxyhydroxides is expected to clearly improve the global electrochemical performances of the material at high regime.

\section{Results and Discussion}

\section{Synthesis Strategy}

Stable colloidal suspensions of exfoliated manganese oxide and $\beta 3-\mathrm{CoOOH}$ "building nanoblocks" were obtained through an exfoliation process that is a crucial point in our strategy. As reported in literature, it can be performed starting from various layered $\mathrm{MnO}_{2}$ precursors. $(43,44,61,62)$ In all cases, exfoliation requires placing the lamellar birnessite phases in acidic medium for nearly complete exchange of interlayer ions for protons. Protonated birnessite phases are thus obtained, with typical formulas such as $\mathrm{H}_{0.24}{ }^{+} \mathrm{K}_{0.04}{ }^{+}\left(\mathrm{H}_{2} \mathrm{O}\right)_{0.27} \mathrm{Mn}^{3.56+} \mathrm{O}_{2}$ (in the case of a potassium precursor). They will be denoted as $\mathrm{H}-\mathrm{MnO}_{2}$. The resulting proton intercalated phase is then treated in $\mathrm{TBAOH}$ (tetraalkylammonium hydroxide) for $\mathrm{TBA}^{+}$ions to be intercalated through proton exchange, which results in the swelling of the structure. The concentration of $\mathrm{TBA}^{+}$varies according to the nature of the initial phase (details in Supporting Information), as the quantity of protons in the interlayer space determines the amount of TBA ${ }^{+}$needed. This swelling is a preliminary step followed by sonication/shaking steps that modify the attractive and repulsive forces between slabs, leading to subsequent exfoliation. The exfoliation of cobalt oxides is much less studied than the manganese ones, as the tetravalent cobalt oxidation state is reported to be very unstable throughout the exfoliation process.(63-65) Nevertheless, the exfoliation of $\beta 3$ - $\mathrm{CoOOH}$ phases, with typical formula such as $\left\{\mathrm{H}_{0.86} \mathrm{Na}_{0.06}\left(\mathrm{H}_{2} \mathrm{O}\right)_{0.19}\right\}_{\text {interslab }}\left\{\mathrm{CoO}_{2}\right\}_{\text {slab }}$, was successfully carried out in a similar manner as for $\mathrm{Mn}$. The obtained colloidal suspensions are stable for up to 6 months concerning $\mathrm{H}-\mathrm{MnO}_{2}$ and three months for $\beta 3-\mathrm{CoOOH}$.

To control their restacking, a study of the surface charge of suspended nanosheets(66) is necessary to better understand the exfoliated particles. Indeed, the surface charge is the driving force for a controlled assembly. Depending on the $\mathrm{pH}$ and the oxidation state of metallic ions constitutive of the layers, the surface charge of the layers of interest can be clearly known through zeta potential measurements, and $\mathrm{pH}$ domains have been identified in which the objects are negatively or positively charged (as shown in Figure S1 in the Supporting Information). As a result, various restacking methods based on electrostatic interactions between the "building nanoblocks" were determined, as summarized in Figure 1.

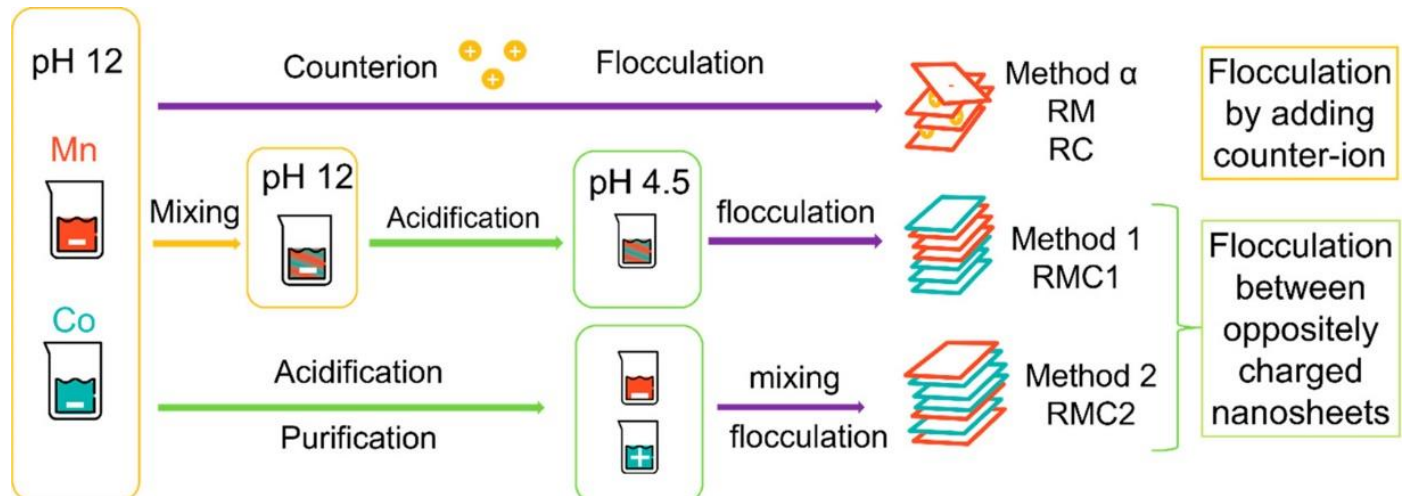

Figure 1. Schematic representation of restacking methods. Method a consists in the destabilization of the initial solutions by addition of a counterion. This method is used for the restacking of monoelement layered materials ("RM" for restacked manganese oxide and "RC" for restacked cobalt oxide). Method 1 consists of mixing the suspensions at high $\mathrm{pH}=12$ then lowering it down, yielding the RMC1 material (restacked manganese-cobalt material). Method 2 consists of lowering the $\mathrm{pH}$ of each suspension before mixing it, yielding the RMC2 material.

The interest of the exfoliation/restacking strategy toward electrochemical properties is first validated by studying the restacking of monoelement (Mn or Co) "building blocks". Only then do we proceed to the preparation of mixed Mn-Co restacked materials. For this, various restacking methods that rely on different interactions between the Mn and Co "building blocks" are used. As a result, the distinct restacked materials present different morphologies and physical chemical properties but always lead to improved electrochemical performances compared to initial materials. In addition, we discuss the influence of the restacking technique and the impact of morphology on the electrochemical performances. To our knowledge, it is the first time that such an extensive 
study on the electrochemical performances of composite materials obtained by exfoliation/restacking of different layered materials has been conducted.

\section{Method $\alpha$}

First, in order to study the effect of the exfoliation/restacking process on the materials properties, the colloidal suspensions of cobalt or manganese-based objects are restacked separately using method a. At the high pH of $\mathrm{Mn}$ and Co-based colloidal suspensions after exfoliation (without further treatment) $(\mathrm{pH}=12)$, the surface charge of the Mn and Co-based particles is negative (see Figure S1 in the Supporting Information). Therefore, the most direct restacking technique is to induce flocculation of the negatively charged nanosheets by increasing the ionic strength of the solution, thus lowering the screening effect between slabs. This flocculation does not affect the 2D morphology of the nanosheets and usually produces a randomly restacked lamellar aggregate. It is performed by adding dropwise a solution of $1 \mathrm{M} \mathrm{NaCl}$ into the stable suspension until visible aggregation. RM (respectively RC) corresponds to the material obtained after exfoliation/restacking of $\mathrm{H}-\mathrm{MnO}_{2}$ (respectively $\beta 3-\mathrm{CoOOH}$ ).

\section{Methods 1 and 2}

Mixed manganese-cobalt materials are obtained by inducing flocculation between oppositely charged nanosheets. Actually, initial suspensions are both negatively charged. However, by shifting the pH to 4.5, Co-based nanosheets become positively charged and can attract negative Mn-based nanosheets. Two different strategies to change the $\mathrm{pH}$ can be adopted, leading to the RMC1 and RMC2 restacked Mn-Co materials. A schematic summary of the restacking methods is presented in Figure 1 (methods 1 and 2). First, method 1 consists of mixing both initial colloidal suspensions (i.e., at the exfoliation $\mathrm{pH}$ of 12), leading to a mixed stable colloidal suspension. Then, in order to induce flocculation, the $\mathrm{pH}$ is decreased from 12 to the optimum $\mathrm{pH}$ of 4.5 by addition of nitric acid. A visual flocculation is observed, and the deposit is centrifuged and dried to obtain RMC1 (method 1). The second restacking method consists of bringing the $\mathrm{Mn}$ and Co-based colloidal suspensions to the optimum $\mathrm{pH} 4.5$ separately, before mixing them together in a third vessel while carefully controlling the $\mathrm{pH}$. This leads to RMC2 (method 2). The main difficulty of this last strategy is to stabilize the initial suspensions at $\mathrm{pH} 4.5 \mathrm{before}$ mixing. Indeed, as the $\mathrm{pH}$ is lowered, ionic strength increases, leading to destabilization of the suspensions that aggregate. To counter this effect, purification is necessary with multiple cycles of centrifugation and resuspension by ultrasonication prior to the restacking in order to get a finer restacking. At the end of these exfoliation/restacking processes, the mixed layered metal oxides obtained are analyzed. As a reference sample, mixed layered metal oxides are also prepared by simple thorough grinding, in a mortar, of initial H-MnO2 and $\beta 3-\mathrm{CoOOH}$ pristine powders (before exfoliation). The obtained sample is denoted as MG (mechanically ground). For comparison sake, the Mn:Co ratio is fixed to 1:1 for all the mixed restacked materials, allowing an in-depth study of the influence of the restacking technique.

Before further discussion about the restacking, the successful exfoliation into building nanoblocks has been verified with various microscopy techniques. SEM and TEM images of the pristine birnessite and cobalt oxyhydroxide are presented in Figure $2 \mathrm{a}-\mathrm{d}$. A clear difference in morphology and size between pristine $\mathrm{H}_{-} \mathrm{MnO}_{2}$ and $\beta 3-\mathrm{CoOOH}$ can be observed. Indeed, $\mathrm{H}-\mathrm{MnO}_{2}$ particles are wrinkled, veil-like lamellar objects. Particles do not appear as single objects but are rather aggregated which renders their size difficult to determine.

Nevertheless, the objects are estimated to be hundreds of nanometer wide. Whereas $\beta 3-\mathrm{CoOOH}$ particles exist as flat hexagonal platelets with clear distinct edges. They are very well-defined; the platelets are around $60 \mathrm{~nm}$ wide and $10 \mathrm{~nm}$ thick.

The effect of the exfoliation process on the object morphology is presented in Figure 2e,f. Exfoliated $\mathrm{H}_{-} \mathrm{MnO}_{2}$ appears as micrometric sheets constituted by few layers, with a folding of the edges that reveals substantial exfoliation. The faint but uniform contrast reveals their ultrathin nature. In the case of cobalt based materials, the TEM image in Figure $2 \mathrm{f}$ shows platelet shapes of approximately $60-70 \mathrm{~nm}$ in good accordance with the morphology observed for the starting phase before exfoliation. Tapping mode AFM was used to precisely measure the thickness of exfoliated objects, in order to estimate the number of oxide layers along the $z$-axis. The images and height profiles presented in Figure $2 \mathrm{~g}$, h correspond to nanosheets collected after purification of the exfoliated colloidal suspensions. 

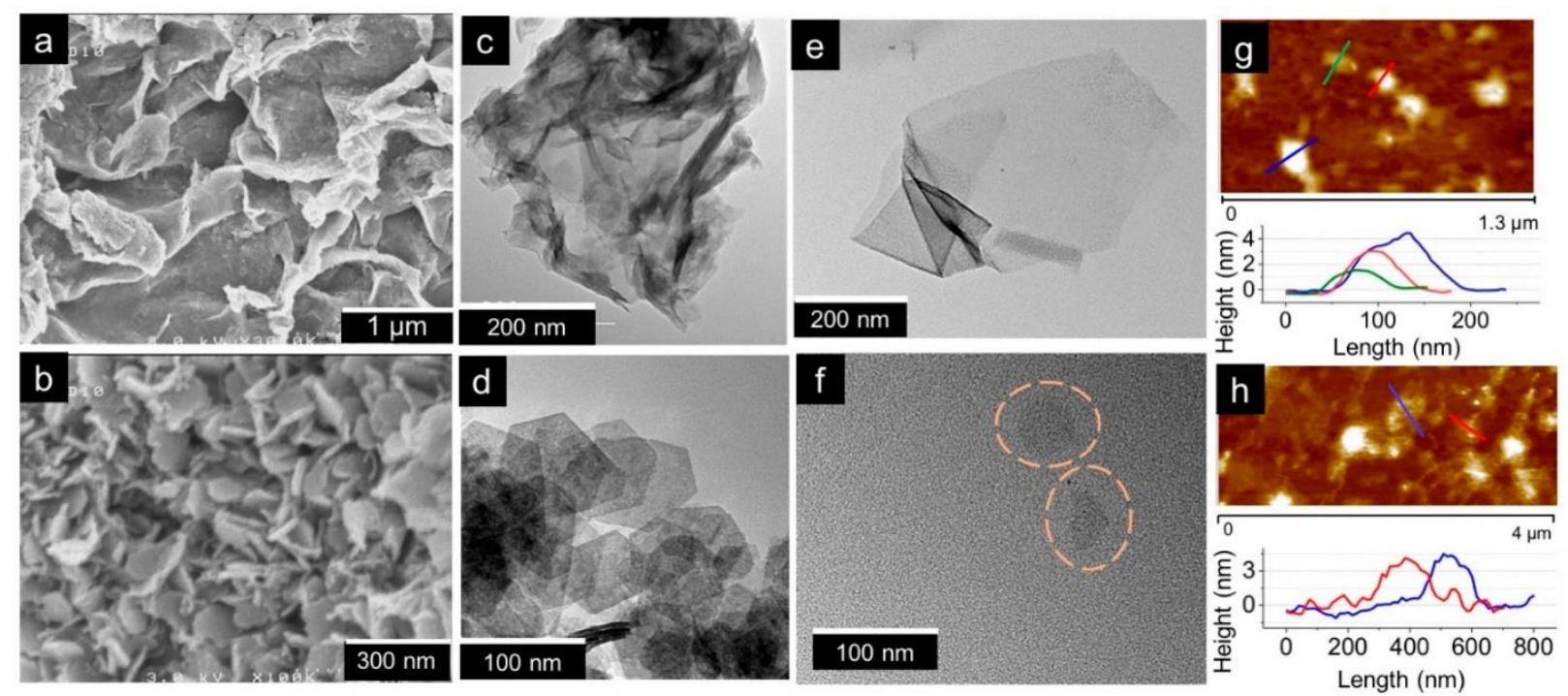

Figure 2. SEM images of (a) pristine birnessite $\mathrm{H}-\mathrm{MnO}_{2}$ and (b) pristine $\beta 3-\mathrm{CoOOH}$. TEM images of (c) pristine birnessite $\mathrm{H}-\mathrm{MnO}_{2}$ and (d) pristine $\beta 3-\mathrm{CoOOH}$. Exfoliated nanosheets of (e) $\mathrm{H}-\mathrm{MnO}_{2}$ and (f) $\beta 3-\mathrm{CoOOH}$. Tapping-mode AFM images and height profiles obtained in the last supernatant (50000 rpm) of exfoliated colloidal suspensions of (g) $\mathrm{H}-\mathrm{MnO}_{2}$ nanosheets and (h) $\beta 3-\mathrm{CoOOH}$ nanosheets.

Exfoliated $\mathrm{H}-\mathrm{MnO}_{2}$ sheets with a minimum thickness of $1.5 \mathrm{~nm}$ can be detected. According to crystallographic data, the theoretical thickness of one layer is $0.38 \mathrm{~nm}$. However, the presence of water molecules between the nanosheets induces a discrepancy between the theoretical thickness and the experimentally obtained value. It can be therefore assumed that the height profile measured corresponds to two or three layers of manganese oxide. In the case of $\beta 3$ $\mathrm{CoOOH}$ exfoliated particles, height profiles reveal the presence of $4 \mathrm{~nm}$ thick particles. From the crystal structure, the thickness of a $\mathrm{CoO}_{2}$ monolayer is $0.48 \mathrm{~nm}$, thus revealing that, considering the hydration of nanosheets, six to eight sheets compose the nanoobjects after exfoliation.

\section{X-ray Diffraction Study}

The X-ray diffraction patterns of the various materials synthesized and of and their parents (respectively $\mathrm{H}-\mathrm{MnO}_{2}$ and $\beta 3-\mathrm{CoOOH})$ are shown in Figure 3. As far as the pure manganese (RM) or cobalt (RC) restacked materials are concerned, their diagrams ( $b$ and $d$ ) can be compared to those of their parent materials ( $a$ and $c)$. The line indexation reported for the manganese materials $\left(\mathrm{H}-\mathrm{MnO}_{2}\right.$ and $\left.\mathrm{RM}\right)$ corresponds to a hexagonal cell (space group $P \overline{3 m} 1$, one slab per cell). For the cobalt-based materials ( $\beta 3-\mathrm{CoOOH}$ and $\mathrm{RC})$, the indexation is performed with three slabs per hexagonal cell (space group $R \overline{3 m}$ ). The sizes of the coherent domains, determined by the Scherrer method, on the basis of the $\left(00^{l}\right)$ lines and (110) lines, are gathered in Table S1 (in the Supporting Information).

Let us remember that the sizes associated with $\left(00^{l}\right)$ (respectively 110$)$ lines correspond to the thickness (respectively width) of the coherent domains in a platelet morphology. Although $\mathrm{RM}$ and $\mathrm{H}-\mathrm{MnO}_{2}$ both present patterns characteristic of birnessite phases, the lines are significantly broadened in the restacked sample, suggesting a significant decrease of the size of coherent domains in the (001) and (110) directions. This is also verified by the broadening of the $(10 l)$ lines observed in the final restacked material, characteristic of turbostraticity. In addition, the inter-reticular distance of the (001) line corresponding to the interslab distance is slightly decreased for $\mathrm{RM}$ as compared with $\mathrm{H}-\mathrm{MnO}_{2}$, indicating the successful intercalation of $\mathrm{Na}^{+}$ions between the Mn-based layers. In comparison, the diffraction patterns of $\mathrm{RC}$ and its precursor $\beta 3-\mathrm{CoOOH}$ are similar (Figure 3c,d), with no significant change in the size of coherent domains. This behavior suggests that the structure and crystallinity of cobalt-based materials are maintained by the exfoliation/restacking process. Flatness and rigidity of the $\beta 3-\mathrm{CoOOH}$ layers revealed on Figure 2 could allow an easier restacking as compared to veil-like H$\mathrm{MnO}_{2}$ nanosheets. 


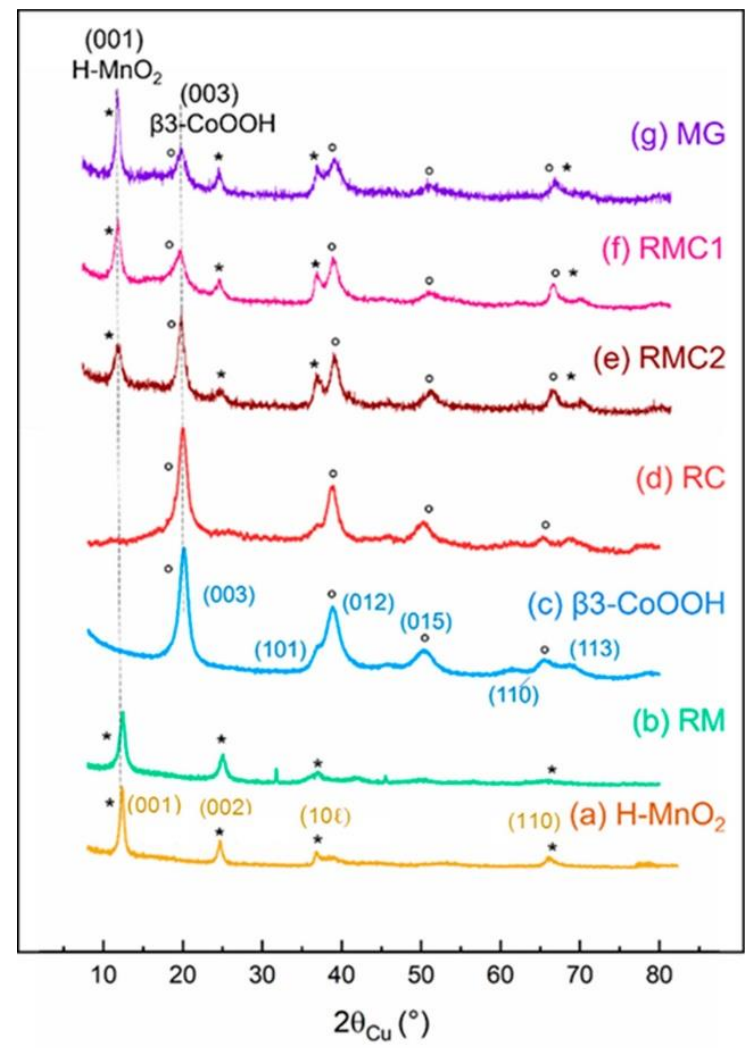

Figure 3. X-ray diffraction patterns of (a) pristine $\mathrm{H}-\mathrm{MnO} 2,(b)$ restacked manganese oxide (RM), (c) pristine $\beta 3-\mathrm{CoOOH}$, (d) restacked cobalt oxide (RC), (e) mixed material obtained by exfoliation/restacking with method 2 (RMC2), (f) mixed material obtained by exfoliation/restacking with method 1 (RMC1), and $(g)$ reference material obtained by mechanical grinding of pristine materials (MG). The lines that correspond to $\mathrm{H}-\mathrm{MnO} 2$ ( $33-\mathrm{CoOOH}$ respectively) are marked with (*) ((deg) respectively).

Mixed Mn-Co restacked materials RMC1 and RMC2 as well as the material prepared by mechanical grinding MG exhibit strong similarities in their X-ray diffraction patterns (Figure 3e-g). The Mn:Co ratios have been verified by chemical analysis, and all present a 1:1 ratio. The three diffractograms present lines characteristic of a mixture of the initial two phases: $\mathrm{H}-\mathrm{MnO}_{2}$ and $\beta 3-\mathrm{CoOOH}$. This suggests a successful recombination of initial manganese and cobalt "building blocks" through exfoliation and various restacking processes. However, depending on the restacking method, the width and intensity of the (001) line of birnessite and of the (003) line of the cobalt oxyhydroxide phase vary, revealing different nanostructures. The intensity ratio between the (001) line of birnessite and the (003) line of the cobalt phase is in favor of birnessite for RMC1 obtained by fast acidification of the mixed colloidal suspensions. On the contrary, when the acidification is performed before mixing (RMC2), the intensity ratio is in favor of the cobalt phase.

The average crystallite sizes along the $\left(00^{l}\right)$ directions (thickness of platelet-like objects), determined by the Scherrer formula, for manganese oxide and cobalt oxyhydroxide are gathered in Table S2 in the Supporting Information. The thickness of the manganese-based domains seems to be quite constant (around 6-7 nm) whatever the restacking method, while the thickness of the cobalt-based domains is higher for RMC2 $(6 \mathrm{~nm})$ than for RMC1 (3 nm), suggesting that restacking kinetics plays a key role on the nanostructure of the final composite.

\section{Scanning and Transmission Electron Microscopy Study}

The organization at the mesoscopic scale of the mixed materials has been probed by SEM-EDS and TEM-EDS (energy-dispersive X-ray spectroscopy). The SEM images (presented in Figure 4) show that, in spite of the same $\mathrm{Mn}:$ Co ratio (determined by ICP analysis), the topographies of the mixed materials are very different. 

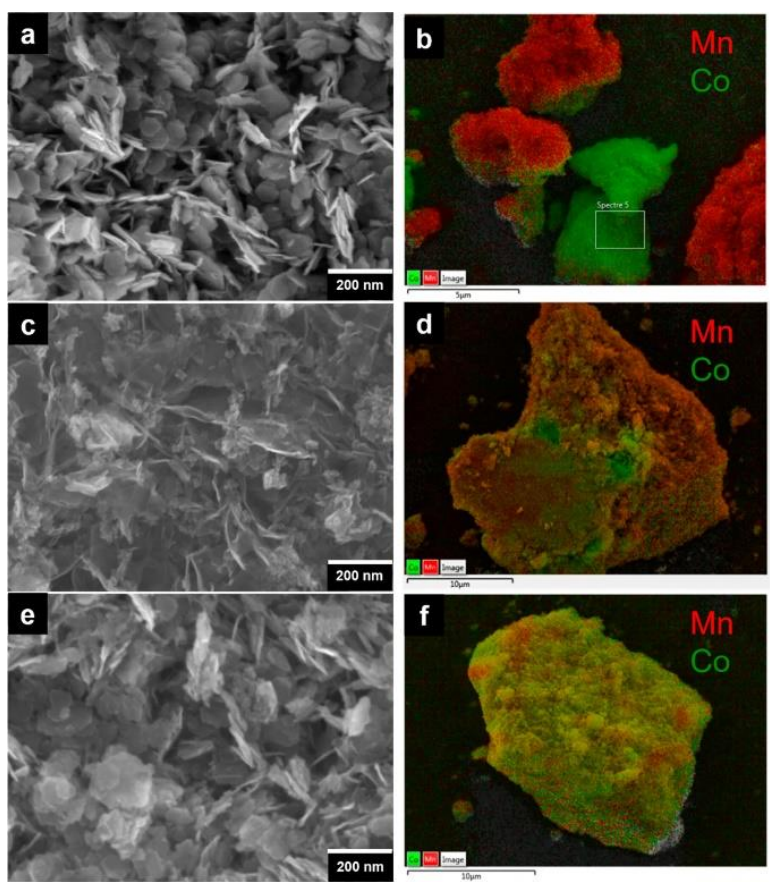

Figure 4. ( $a$ and b) SEM-EDS images of MG composite obtained by mechanical grinding of H-MnO2 and $\beta 3-\mathrm{CoOOH}$. (c and d) SEM-EDS images of RMC1 (restacking according to method 1). (e and f) SEM-EDS images of RMC2 (restacking according to method 2). Red and green colors correspond to manganese-rich and cobalt-rich, respectively, areas.

The $\mathrm{H}-\mathrm{MnO}_{2}$ oxide particles are hardly distinguishable because of their veil-like morphologies, when the hexagonal platelets of $\beta 3-\mathrm{CoOOH}$ can be easily observed. Moreover, these hexagonal platelets appear slightly more aggregated in the RMC2 sample. The EDS images of each sample also reveal significant differences. Images of the simply ground material MG show a clear segregation between the cobalt oxyhydroxide particles appearing in green and the manganese oxide particles in red, while on the images of the exfoliated/restacked materials RMC1 and RMC2, the Mn and Co elements appear to be intimately mixed, allowing the restacked compounds to be considered as nanocomposites of manganese oxide and cobalt oxyhydroxide. A closer observation of the EDS images of RMC1 and RMC2 leads to the conclusion that the restacking method clearly affects the homogeneity of the manganese and cobalt mixture. RMC2 seems to be the most homogeneous, as revealed by the appearance of a homogeneous yellow color, whereas RMC1 shows more manganese on the surface, the red color being predominant. According to the TEM-EDS images, shown in Figure S2 (in the Supporting Information), it can also be observed that the MG sample shows a clear separation between cobalt oxyhydroxide and manganese oxide phases, whereas RMC1 and RMC2 both present intimately mixed Mn and Co elements. At this scale, it seems like RMC1 is more homogeneous than RMC2, as suggested by the presence of yellow color. However, this is not incoherent with the SEM results, as TEM probes the entire volume of the sample, instead of only the surface. The particle observed in Figure S2b is most likely thicker than the particle observed in Figure S2c.

Although all three mixed materials have the same Mn:Co ratio, their morphologies exhibit differences. This can be reasonably attributed to the restacking technique: the flocculation kinetics are different for Co oxyhydroxides and Mn oxides, depending on the restacking and the $\mathrm{pH}$ modification process used. Indeed, throughout exfoliation, it has been observed that the cobalt oxyhydroxide suspensions are less stable than the manganese oxide suspensions. When $\mathrm{pH}$ is decreased close to the isoelectric point (IEP) of the exfoliated materials, the nanosheets are greatly destabilized and are more inclined to restack one onto another. By rapidly adding the mixed Mn-Co colloidal suspension to a strong acid solution at $\mathrm{pH} 1$, close to the (IEP) of manganese objects, restacking method 1 favors the flocculation of Mn oxides. In the restacking method 2, each colloidal suspension is acidified to $\mathrm{pH} 4.5$ before being mixed together. At this $\mathrm{pH}$, both materials bear a lower surface charge than at high $\mathrm{pH}$, allowing a slight restacking of Co oxyhydroxides. This consequently leads to bigger Co oxyhydroxide crystallites than in method 1, where Co oxyhydroxides are destabilized in the presence of Mn oxide particles and thus restack upon them. The bigger size of cobalt oxyhydroxide crystallites obtained by method 2 is in good agreement with the fact that the size of the coherent domains deduced from the Scherrer method is significantly higher for method 2 (6 $\mathrm{nm}$ ) than for method 1 (3 nm), as reported in Supporting Information Table S2. This size difference is reflected in the X-ray diagrams (Figure 3e,f) by the fact that the line (003) of $\mathrm{CoOOH}$ is thinner and more intense in RMC2 than in RMC1. Restacking method 2 is suitable to obtain Mn-rich and Co-rich aggregates with a similar size, giving rise to the most homogeneous sample. 


\section{Specific Surface Measurements}

As stated before, the electrochemical properties of electrode materials greatly depend on their specific surface area and electronic conductivity. The specific surface area of restacked materials RC, RM, RMC1, and RMC2, obtained by BET measurements, are tabulated in Table 1.

\section{Table 1. Specific Surface Area of Pristine Materials and Restacked Materials with Ratio of Mn:Co of 1:1 Obtained by Different Restacking Techniques}

\begin{tabular}{lc}
\multicolumn{1}{c}{ sample } & specific BET surface $\left(\mathrm{m}^{2} / \mathrm{g}\right)$ \\
$\mathrm{H}-\mathrm{MnO}_{2}$ & 85 \\
$\mathrm{RM}$ & 30 \\
$\beta 3-\mathrm{CoOOH}$ & 110 \\
$\mathrm{RC}$ & 45 \\
$\mathrm{MG}$ & 95 \\
$\mathrm{RMC} 1$ & 92 \\
$\mathrm{RMC2}$ & 100 \\
\hline
\end{tabular}

From the $\mathrm{N}_{2}$ adsorption/desorption isotherms (Figure $\mathrm{S} 3$ of the Supporting Information), it seems that simple restacked materials RM and RC exhibit lower specific surface area than their parent materials. Intuitively, it is expected that the surface area should increase after exfoliation. A possible explanation for this decrease is the restacking strategy employed. The restacking process is very random: instead of being well ordered, the nanosheets will tend to restack in arbitrary directions in a more packed manner, which could lead to a decrease of surface area. The isotherms of the mixed restacked RMC1 and RMC2 materials and of reference mechanically mixed MG exhibit a similar shape, as pristine materials. The calculated specific surfaces of RMC1, RMC2, and MG are also very close (between 90 to $100 \mathrm{~m}^{2} / \mathrm{g}$ ) and intermediate between pristine manganese oxide $\left(85 \mathrm{~m}^{2} / \mathrm{g}\right)$ and cobalt oxyhydroxide $\left(110 \mathrm{~m}^{2} / \mathrm{g}\right)$. At first sight, it appears surprising that the exfoliation/restacking process results in surface area decrease only for RM or RC and not for RMC. As a matter of fact, the restacking processes used for RM (or RC) and RMC are different. The negatively charged nanosheets of RM and RC are restacked by flocculation on $\mathrm{Na}^{+}$cations provided by simple addition of $\mathrm{NaCl}$ in the pristine stable colloidal suspensions, whereas RMC is formed by attraction of positive Co-based nanosheets and negative $\mathrm{Mn}$-based nanosheets at $\mathrm{pH}$ 4.5 , without any salt addition. It can be supposed that, for RM and RC, the flocculation of identical Mn or Co slabs on $\mathrm{Na}^{+}$cations is likely to be performed in a more packed manner, while in RMC samples, two different types of sheets are restacked, which probably does not favor such a packed sample.

\section{Electronic Conductivity Measurements}

The electronic conductivity measurement results of RMC2 (most homogeneous mixed restacked sample) and MG (mechanical ground mixture) are presented and compared to the pristine materials in Figure 5. H-MnO2 presents a low electronic conductivity that is activated by temperature. At room temperature, the conductivity value is equal to $7.2 \times 10^{-5} \mathrm{~S} / \mathrm{cm}$, while $\beta 3-\mathrm{CoOOH}$ exhibits a good conductivity of $1.8 \mathrm{~S} / \mathrm{cm}$, which is 5 orders of magnitude higher. For a composite made of $50 \% \mathrm{H}-\mathrm{MnO} 2$ and $50 \% \beta 3-\mathrm{CoOOH}$, in which $\beta 3-\mathrm{CoOOH}$ particles should percolate, an intermediate conductivity would be expected. Nevertheless, in the case of a simple mechanical grinding $\left(1.4 \times 10^{-5} \mathrm{~S} / \mathrm{cm}\right)$ as well as of restacking $\left(6.7 \times 10^{-5} \mathrm{~S} / \mathrm{cm}\right)$, the conductivities of the samples are on the same order of magnitude as $\mathrm{H}-\mathrm{MnO}_{2}\left(7.2 \times 10^{-5} \mathrm{~S} / \mathrm{cm}\right)$. We do not observe the expected increase of electronic conductivity due to the presence of $\beta 3-\mathrm{CoOOH}$ at the sample scale. Nevertheless, as it will be reported in a forthcoming paper specifically devoted to electronic properties, BDS (broadband dielectric spectroscopy) measurements carried out on our materials help explain this observation. The results lead to the conclusion that the global conductivity of the Mn-Co composite is not enhanced because there is no electronic percolation between the Co nano-objects, in spite of a geometrical percolation. This can be attributed to an electron trapping phenomenon occurring at particle junctions. $(67,68)$ The most probable hypothesis is that the electrons are blocked on the grain boundaries by the various protons present in the structure. Their poor conductivity could have a 
detrimental impact on the electrochemical properties of the restacked materials, especially on RMC2, the most homogeneously restacked material.

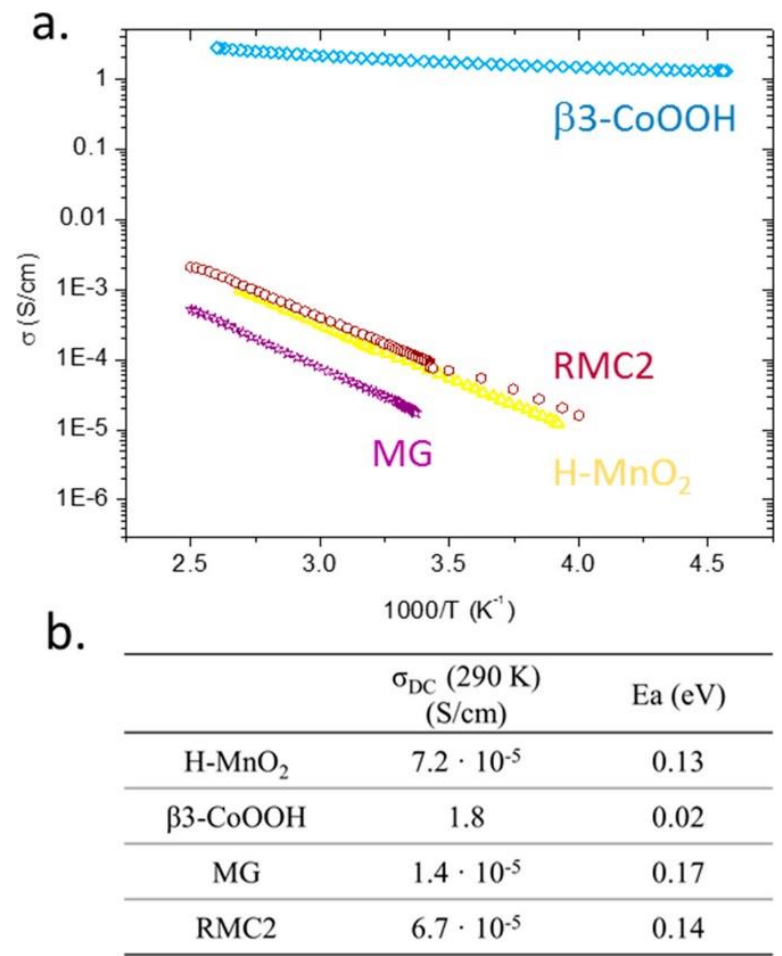

Figure 5. (a) Thermal variation of electronic conductivity for the restacked material RMC2 and the MG sample obtained by mechanical grinding of $\mathrm{H}-\mathrm{MnO} 2$ and $\beta 3-\mathrm{CoOOH}$ (ratio $50 \% \mathrm{Mn}$ and $50 \%$ Co). These are compared to the as-prepared pristine materials. (b) Table presenting activation energy and conductivity at room temperature of the materials.

\section{Electrochemical Properties}

The electrochemical properties of the materials are illustrated by the curves in Figure 6. Impact of exfoliation on electrochemical properties was first evaluated by comparing the electrochemical behaviors of exfoliated/flocculated manganese oxide (RM) and cobalt oxyhydroxide (RC) to their pristine parents $\mathrm{H}-\mathrm{MnO}_{2}$ and $\beta 3-\mathrm{CoOOH}$. The variations of specific capacitances vs scan rate are presented in Figure $6 \mathrm{a}$ for the four materials.

Pristine $\beta 3-\mathrm{CoOOH}$ reveals a very low capacitance while pristine $\mathrm{H}-\mathrm{MnO}_{2}$ exhibits a high pseudocapacitance, as already reported.(69,70) In both cases, the specific capacitances obtained for exfoliated/restacked materials are systematically higher than their initial parents. This trend can be attributed to a different structuration of nanosheets induced by exfoliation, resulting in an increase of the active electrochemical surface in contact with the electrolyte. However, as shown previously, the specific surface areas are significantly lower for the exfoliated/restacked materials than for the initial materials. This is contrary to what is expected in relation with the enhanced specific capacitances. It is possible to interpret this by claiming the discrepancy between specific surface area measured by BET and the electrochemically active surface that contributes to charge storage. In other terms, for RM or RC, the number of electrochemically active sites might increase due to the exfoliation effect, whereas the surface accessible by gas decreases (measured by BET) due to special reorganization of the nanosheets during flocculation on $\mathrm{Na}^{+}$cations. 
a.

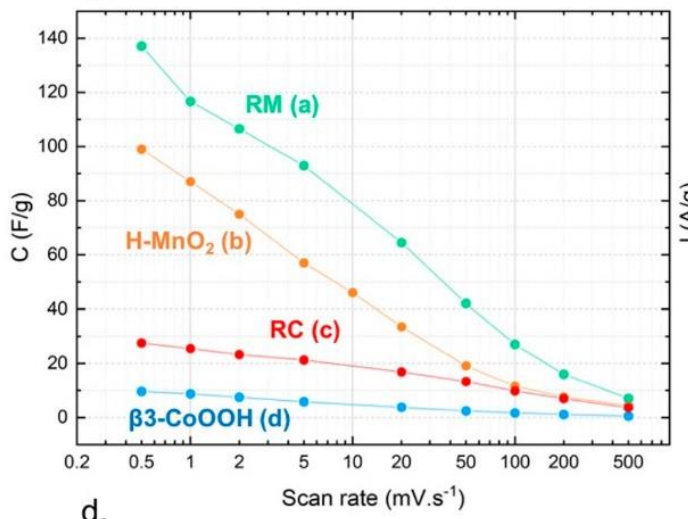

d.

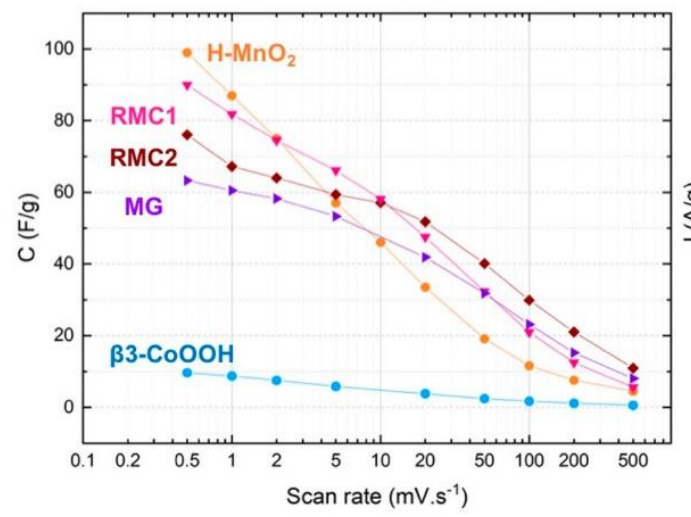

b.

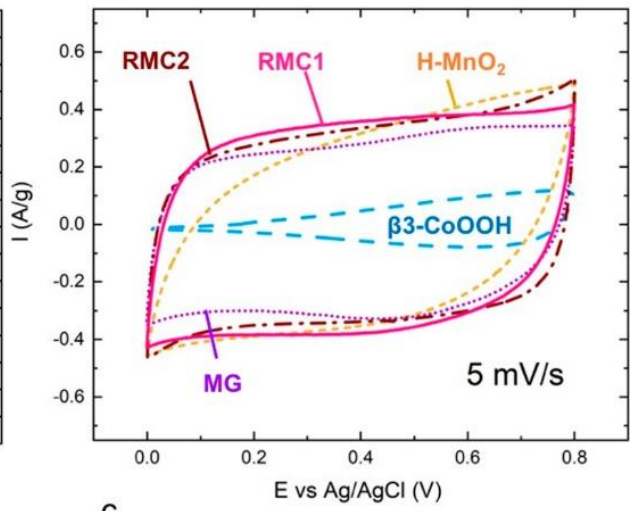

c.

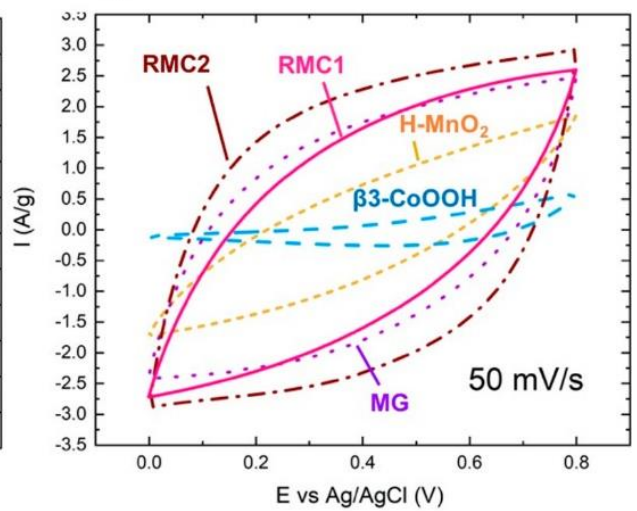

Figure 6. (a) Capacitance (normalized to the mass of active material) vs scan rate obtained for initial materials and corresponding exfoliated/restacked derived materials prepared by flocculation (method $\alpha$ ). Cyclic voltammograms of initial $\beta 3-\mathrm{CoOOH}, \mathrm{H}-\mathrm{MnO}_{2}$, compared to mechanically ground mixture $\mathrm{MG}$, and restacked materials RMC1 and RMC2 in a three-cell configuration, with $\mathrm{Ag} / \mathrm{AgCl}$ as reference electrode and Pt as counter electrode, in $0.5 \mathrm{M} \mathrm{K}_{2} \mathrm{SO}_{4}$ electrolyte at scan rates of (b) $5 \mathrm{mV} / \mathrm{s}$ and (c) 50 $\mathrm{mV} / \mathrm{s}$. (d) Capacitance vs scan rate obtained for all the materials.

After proving that simple exfoliation efficiently improves the electrochemical performances as compared to pristine initial materials, it is interesting to test restacked mixed manganese-cobalt materials (RMC1, RMC2) as supercapacitor electrodes, in comparison with the simple mechanical mixture of the pristine materials (MG). The cyclic voltammograms are presented in Figure $6 \mathrm{~b}, \mathrm{c}$, and the variations of the corresponding specific capacitances are displayed in Figure 6d. At a scan rate of $5 \mathrm{mV} / \mathrm{s}$, the voltammograms of all three composite samples exhibit more rectangular shapes than initial birnessite, which means that they present lower internal resistance than the initial materials. Moreover, since these materials contain only $50 \%$ of birnessite as active material, it can be concluded that the enhanced diffusion pathways allow a better use of birnessite pseudocapacitance. Nevertheless, at a low rate (lower than $10 \mathrm{mV} / \mathrm{s}$ ), the specific capacitances of the three composites are lower than for pristine birnessite. It is at high regime, when the scan rate is increased to $50 \mathrm{mV} / \mathrm{s}$, that the beneficial effect of adding cobalt oxyhydroxide to the manganese oxide active material is the most obvious. Even with the presence of only $50 \%$ of birnessite, capacitances of all mixed materials are higher than the $\mathrm{H}-\mathrm{MnO}_{2}$ initial birnessite. Moreover, RMC2 is revealed to be more performant than RMC1 and has been proved below to be more homogeneous than RMC1, thus indicating the impact of the restacking method on the performances of the obtained materials. Such a mixture at the nanometer scale favors charge carrier transport in the materials, even if no significant increase of the electronic conductivity measured by the four probe method can be detected. To sum up, restacking method 2 consisting of first adjusting the $\mathrm{pH}$ of separate colloidal suspensions of $\mathrm{Mn}$ and Co-based nanosheets before mixing them together enables a finer, more homogeneous flocculation leading to the most performant material of the series.

In the context of an application in supercapacitors, the cyclability of the electrodes is very important. RMC2 was then cycled up to 10000 cycles (cyclic voltammetry at $10 \mathrm{mV} / \mathrm{s}$ ) and compared to its mechanically ground homologous material (MG). The capacitance variations vs cycle number are shown in the Supporting Information (Figure S4). First of all, the restacked material shows higher specific capacitance than the simply mixed one, even at the very beginning of the cycling. Second, its capacitance is constant with a high capacitance retention up to 
10000 cycles, whereas the MG mechanically mixed material shows a non-negligible decrease of capacitance after 3000 cycles. The bad cyclability of the simply mixed composite can be attributed to a progressive loss of cohesion between the manganese oxide and carbon particles during cycling, leading to higher intergranular resistances, whereas the interfaces between the manganese and cobalt particles might be more robust in the restacked sample.

\section{Conclusion}

The results presented here illustrate the success of the exfoliation/restacking strategy of 2D layered materials in designing mixed nanocomposites with enhanced electrochemical performances. Starting from the 2D layered birnessite, which presents excellent pseudocapacitive properties but low conductivity, we address this issue with the help of cobalt oxyhydroxide, known for its high electronic conductivity. In order to preserve the properties of each initial material while creating a synergistic effect, the synthesis involves delamination into "building nanoblocks" which are then restacked in order to obtain a novel nanocomposite material. Two restacking processes were conducted, and in both cases, the obtained mixed compounds are identified as Mn-Co nanocomposites showing a more intimate mixture at the nanometric scale than by mechanical grinding of pristine materials. As far as the electrochemical properties are concerned, a synergistic effect between Mn oxides and Co oxyhydroxides is achieved, and a clear impact of the restacking method on the morphology and on the electrochemical activity is observed. Deeper influence of the Mn:Co ratio in such nanocomposites is presently investigated and will be the object of a forthcoming paper.

\section{Methods}

\section{Preparation of Initial 2D Nanomaterials}

Layered potassium manganese oxide powder was prepared at room temperature by precipitation of a manganese salt in an alkaline oxidant solution. A total of $10.0 \mathrm{mmol}$ of $\mathrm{Mn}\left(\mathrm{SO}_{4}\right) \cdot \mathrm{H}_{2} \mathrm{O}$ (Sigma-Aldrich) was dissolved in $30 \mathrm{~mL}$ of water in a plastic beaker, and a solution of $\mathrm{KOH}$ (Sigma-Aldrich, $6 \mathrm{M}, 30 \mathrm{~mL}$ ) was added rapidly, leading to a brown solution. The vigorous stirring was maintained while the $7.0 \mathrm{mmol}$ of $\mathrm{K}_{2} \mathrm{~S}_{2} \mathrm{O}_{8}$ (Sigma-Aldrich) was added very slowly over a period of over $45 \mathrm{~min}$. The resulting brown-black slurry was stirred for an additional $30 \mathrm{~min}$ before being washed with distilled water and finally dried at $60^{\circ} \mathrm{C}$ for $24 \mathrm{~h}$. To facilitate exfoliation, the resulting powder was treated with $0.1 \mathrm{M} \mathrm{HCl}$ solution for 4 days at room temperature to produce the proton-inserted birnessite manganese oxide $\left(\mathrm{H}-\mathrm{MnO}_{2}\right)$. The exfoliated layered manganese oxide nanosheets were synthesized by reaction of the protonated manganese birnessite with tetrabutylammonium hydroxide (Sigma-Aldrich) for 12 days at room temperature. The amount of TBAOH applied with respect to the exchangeable protons $\left(\mathrm{TBA}^{+} / \mathrm{H}^{+}\right)$ was fixed to 10 after optimization. The resulting colloidal suspension was centrifuged at a speed of $6000 \mathrm{rpm}$ for $15 \mathrm{~min}$ to remove the fraction of incompletely exfoliated particles.

$\beta$ (III)-type layered cobalt oxyhydroxide was synthesized by precipitation at room temperature of a cobalt salt in alkaline oxidant media. $\mathrm{Co}\left(\mathrm{NO}_{3}\right)_{2}$ (Sigma-Aldrich) was dissolved in $300 \mathrm{~mL}$ of distilled water and added dropwise in $11 \mathrm{~mL}$ of $2 \mathrm{M} \mathrm{NaOH}$ (Sigma-Aldrich). Then, a solution of $4.8 \mathrm{M}-\mathrm{NaClO}$ was added as a strong oxidant. The mixture was stirred for 5-30 min and centrifugated 8 times (6000 rpm for $4 \mathrm{~min}$ ) until the supernatant reached neutrality. The powder was recovered after having dried at $60^{\circ} \mathrm{C}$ for $24 \mathrm{~h}$. The powder was suspended in an aqueous solution of TBAOH for 20 days at room temperature under vigorous stirring. The amount of TBAOH applied with respect to the exchangeable protons (TBA+/H+) was fixed to 2 after optimization. Then the colloidal suspension obtained was centrifuged at $6000 \mathrm{rpm}$ for $15 \mathrm{~min}$ to remove the fraction of incompletely exfoliated particles.

\section{Material Characterization}

X-ray Diffraction

The X-ray powder diffraction analyses were carried out on a Philips Panalytical X'Pert Pro diffractometer with a Bragg-Brentano $\theta-\theta$ geometry. In the case of manganese oxides, a copper Ka radiation was used $\left(\lambda_{\mathrm{Ka} 2}=1.54439 \AA\right.$, $\lambda_{K a 1}=1.54056 \AA$ ). The powder diffraction patterns were recorded for about $2 \mathrm{~h}$ in the $8^{\circ}-80^{\circ}(2 \theta)$ angular range, with a $0.0167^{\circ}(2 \theta)$ step size and a $2.122^{\circ}(2 \theta)$ active width in the detector. In the case of cobalt oxyhydroxides, a cobalt Ka radiation $\left(\lambda_{\mathrm{Ka} 2}=1.7928 \AA, \lambda_{\mathrm{Ka} 1}=1.7889 \AA\right)$ was used. The powder diffraction patterns were recorded for about $10 \mathrm{~h}$ in the $10^{\circ}-110^{\circ}(2 \theta)$ angular range, with a $0.0167^{\circ}(2 \theta)$ step size and a $2.122^{\circ}(2 \theta)$ active width in the detector. 


\section{ICP-OES Spectroscopy Measurements}

$\mathrm{Co}, \mathrm{Mn}, \mathrm{K}$, and Na elements were quantified in our samples using a Varian 720ES ICP-OES spectrometer.

Powdered samples (10-20 mg) were dissolved into boiling $\mathrm{HCl}$, and the obtained solutions were diluted in order to obtain concentrations varying from 1 to $200 \mathrm{mg} / \mathrm{L}$. Solutions were then introduced in a nebulization chamber along with an argon flow to create an aerosol.

\section{Transmission Electron Microscopy}

Transmission electron microscopy (TEM) of 2D materials deposited on a carbon-coated copper grid was done with a JEOL 2200FS microscope equipped with a high-resolution camera. The microscope was used at $200 \mathrm{kV}$. In this case, the resolution is $0.19 \mathrm{~nm}$. A JEOL JSM 7800F Prime scanning electron microscope (SEM) equipped with an EDS system was used to characterize the microstructure of the layered materials. The acceleration voltage used was from 1 to $30 \mathrm{kV}$.

Atomic Force Microscopy

Atomic force microscopy images were recorded by the tapping-mode phase imaging using a standard silicon cantilever (approx $20 \mathrm{~N} / \mathrm{m}, 150 \mathrm{kHz}$ ) on a commercial ICON AFM (Bruker). The software used to analyze the images is Nanoscope Analysis. For the analysis, it is particularly critical to avoid reaggregation of nanosheets on the wafer during solvent evaporation. For this, very dilute dispersions are used and drop-casted onto a microscope slide. The slide is pretreated with a freshly cleaved mica surface in order to obtain an atomically flat surface. The zeta potentials of the dispersions were measured with a Malvern Zetasizer Nano series. The Zetasizer Nano series calculates the zeta potential by determining the electrophoretic mobility and then applying the Henry equation. The electrophoretic mobility is obtained by performing an electrophoresis experiment on the sample and measuring the velocity of the particles using laser doppler velocimetry (LDV).

Purification of the Exfoliated Colloidal Suspensions for AFM Measurements on Nanosheets

In order to characterize the produced nanosheets after exfoliation, they must be separated from the bulk unexfoliated material, and size-selection is required. To do so, liquid cascade centrifugation is used, which consists of a multistep process where various centrifugations at increasing speeds (4000, 20000, and $50000 \mathrm{rpm}$ ) are carried out. The first centrifugation at $4000 \mathrm{rpm}$ allows the nonexfoliated material to be recovered in the bottom of the flask. After each subsequent step, size-selected nanosheets are collected as sediments while the supernatant is subjected to another centrifugation at higher rotation speed.

$\mathrm{N}_{2}$ Adsorption Measurements

All the specific surface measurements and characterization of material mesoporosity were conducted by $\mathrm{N}_{2}$ adsorption at $77 \mathrm{~K}$. The measurements were performed on ASAP2010 (Micromeritics Corp., Norcross, GA, U.S.A.). Each sample was degassed at $100^{\circ} \mathrm{C}$ under vacuum during the necessary time to obtain a constant pressure of $10 \mu \mathrm{m} \mathrm{Hg}$ (approximately 12-15 h). The BET model was applied for relative pressures $\left(P / P_{0}\right)$ between 0.01 and 0.3 to calculate the specific surface $S_{\mathrm{BET}}$. Pore distribution was determined using the BJH model. The pore dimensions were calculated from the desorption isotherms.

Electric Conductivity Measurements

The electric conductivity measurements have been performed with the four-probe technique, using direct current in the $230-400 \mathrm{~K}$ temperature range. Because of their instability beyond $400 \mathrm{~K}$, the materials could not be sintered and the measurements were performed on pellets $(8 \mathrm{~mm}$ in diameter) obtained by compacting $200 \mathrm{mg}$ of powder at $1.2 \mathrm{GPa}$.

Electrochemical Cycling

All electrochemical performances were evaluated in a three-electrode cell filled with an $0.5 \mathrm{M} \mathrm{K}_{2} \mathrm{SO}_{4}$ electrolyte. Platinum was used as counter electrode and $\mathrm{Ag} / \mathrm{AgCl}$ as reference electrode. The working electrode was prepared by mixing $80 \%$ active material powder, 5\% PTFE (Sigma-Aldrich, $60 \mathrm{wt} \%$ water suspension), and 15\% of carbon black (conductive additive made of acetylene, 100\% compressed, Alfa Aesar) with ethanol. The mixture was set as an autosupported film, in which an $8 \mathrm{~mm}$ diameter disc was cut and pressed on a stainless steel grid as current collector at $6 \mathrm{t} / \mathrm{cm}^{2}$ for $5 \mathrm{~min}$. Electrochemical cyclic voltammetry was completed using a VMP3 potentiostat (Biologic) under ECLab software and was performed at 5 and $50 \mathrm{mV} / \mathrm{s}$ between 0 and $0.8 \mathrm{~V}$.

\section{Acknowledgements}

This work was supported by RS2E through Celine Tang's grant (LABEX STORE-EX ANR-10-LABX-01). The authors would like to deeply thank Philippe Legros et Marion Gayot from PLACAMAT (UMS 3626 CNRS-UB) for the SEM-EDS and TEM-EDS cartography, respectively. A special notice to Gregory Lefèvre for his help in understanding the interactions between oxides in solution, as well as P. Dagault, C. Denage, and S. Goma for their technical support. 


\section{Supporting informations}

The Supporting Information is available free of charge on the ACS Publications website at DOI:

10.1021/acsaem.9b01263.

Experimental zeta potential-pH curves of initial protonated birnessite $\mathrm{H}-\mathrm{MnO}_{2}$ particles (red) and $\beta 3-\mathrm{CoOOH}$ cobalt oxyhydroxide, average sizes $(\mathrm{nm})$ of coherent domains along $(00 l)$ and $(110)$ directions determined by Scherrer method for pristine and restacked materials, TEM-EDS images of the materials, $\mathrm{N}_{2}$ adsorption/desorption isotherms at $77 \mathrm{~K}$ of the pristine and restacked materials, and cyclability of restacked material RMC2 vs mechanically mixed composite ( $\underline{\mathrm{PDF}})$.

\section{References}

1 Kousksou, T.; Bruel, P.; Jamil, A.; El Rhafiki, T.; Zeraouli, Y. Energy Storage: Applications and Challenges. Sol. Energy Mater. Sol. Cells 2014, 120, 59-80, DOI: 10.1016/j.solmat.2013.08.015

2 Tarascon, J.-M.; Armand, M. Issues and Challenges Facing Rechargeable Lithium Batteries. Nature 2001, 414 (6861), 359- 367, DOI: $10.1038 / 35104644$

3 Conway, B. E. Transition from "supercapacitor" to "Battery" Behavior in Electrochemical Energy Storage. J. Electrochem. Soc. 1991, 138 (6), 1539- 1548, DOI: 10.1149/1.2085829

4 Simon, P.; Gogotsi, Y.; Dunn, B. Where Do Batteries End and Supercapacitors Begin?. Science 2014, 343 (6176), 1210- 1211, DOI: 10.1126/science.1249625

5 Miller, J. R.; Simon, P. Electrochemical Capacitors for Energy Management. Science 2008, 321 (5889), 651- 652, DOI:

10.1126/science.1158736

6 Pandolfo, A. G.; Hollenkamp, A. F. Carbon Properties and Their Role in Supercapacitors. J. Power Sources 2006, 157 (1), 11- 27, DOI: 10.1016/j.jpowsour.2006.02.065

7 Lewandowski, A.; Olejniczak, A.; Galinski, M.; Stepniak, I. Performance of Carbon-Carbon Supercapacitors Based on Organic, Aqueous and Ionic Liquid Electrolytes. J. Power Sources 2010, 195 (17), 5814- 5819, DOI: 10.1016/j.jpowsour.2010.03.082

8 Qu, D. Studies of the Activated Carbons Used in Double-Layer Supercapacitors. J. Power Sources 2002, 109 (2), 403-411, DOI:

10.1016/S0378-7753(02)00108-8

9 Vivekchand, S. R. C.; Rout, C. S.; Subrahmanyam, K. S.; Govindaraj, A.; Rao, C. N. R. Graphene-Based Electrochemical Supercapacitors. Proc. - Indian Acad. Sci., Chem. Sci. 2008, 120 (1), 9- 13, DOI: 10.1007/s12039-008-0002-7

10 Du, X.; Guo, P.; Song, H.; Chen, X. Graphene Nanosheets as Electrode Material for Electric Double-Layer Capacitors. Electrochim. Acta 2010, 55 (16), 4812-4819, DOI: 10.1016/j.electacta.2010.03.047

11 Kaempgen, M.; Chan, C. K.; Ma, J.; Cui, Y.; Gruner, G. Printable Thin Film Supercapacitors Using Single-Walled Carbon Nanotubes. Nano Lett. 2009, 9 (5), 1872-1876, DOI: 10.1021/nl8038579

12 Yu, D.; Dai, L. Self-Assembled Graphene/Carbon Nanotube Hybrid Films for Supercapacitors. J. Phys. Chem. Lett. 2010, 1 (2), 467-470, DOI: $10.1021 / \mathrm{jz} 9003137$

13 Conway, B. E.; Birss, V.; Wojtowicz, J. The Role and Utilization of Pseudocapacitance for Energy Storage by Supercapacitors. J. Power Sources 1997, 66 (1-2), 1-14, DOI: 10.1016/S0378-7753(96)02474-3

14 Zheng, J. P.; Cygan, P. J.; Jow, T. R. Hydrous Ruthenium Oxide as an Electrode Material for Electrochemical Capacitors. J. Electrochem. Soc. 1995, 142 (8), 2699-2703, DOI: 10.1149/1.2050077

15 De Pauli, C. P.; Trasatti, S. Electrochemical Surface Characterization of IrO2 + SnO2Mixed Oxide Electrocatalysts. J. Electroanal. Chem. 1995, 396, 161- 168, DOI: 10.1016/0022-0728(95)03950-L

16 Liu, K.-C.; Anderson, M. A. Porous Nickel Oxide/Nickel Films for Electrochemical Capacitors. J. Electrochem. Soc. 1996, 143 (1), 124- 130, DOI: $10.1149 / 1.1836396$

17 Zhang, Y.; Hu, Z.; An, Y.; Guo, B.; An, N.; Liang, Y.; Wu, H. High-Performance Symmetric Supercapacitor based on Manganese Oxyhydroxide Nanosheets on Carbon Cloth as Binder-Free Electrodes. J. Power Sources 2016, 311, 121-129, DOI:

10.1016/j.jpowsour.2016.02.017

18 Lee, H. Y.; Goodenough, J. B. Supercapacitor Behavior with KCl Electrolyte. J. Solid State Chem. 1999, 144 (1), 220- 223, DOI:

$10.1006 /$ jssc.1998.8128

19 Toupin, M.; Brousse, T.; Bélanger, D. Charge Storage Mechanism of MnO2 Electrode Used in Aqueous Electrochemical Capacitor. Chem. Mater. 2004, 16 (16), 3184-3190, DOI: 10.1021/cm049649j

20 Holze, R.; Wu, Y. P. Intrinsically Conducting Polymers in Electrochemical Energy Technology: Trends and Progress. Electrochim. Acta 2014, 122, 93- 107, DOI: 10.1016/j.electacta.2013.08.100

21 Rudge, A.; Raistrick, I.; Gottesfeld, S.; Ferraris, J. P. A Study of the Electrochemical Properties of Conducting Polymers for Application in Electrochemical Capacitors. Electrochim. Acta 1994, 39 (2), 273- 287, DOI: 10.1016/0013-4686(94)80063-4

22 Laforgue, A.; Simon, P.; Sarrazin, C.; Fauvarque, J.-F. Polythiophene-Based Supercapacitors. J. Power Sources 1999, 80 (1), 142- 148, DOI: 10.1016/S0378-7753(98)00258-4

23 Rudge, A.; Davey, J.; Raistrick, I.; Gottesfeld, S.; Ferraris, J. P. Conducting Polymers as Active Materials in Electrochemical Capacitors. J. Power Sources 1994, 47 (1-2), 89- 107, DOI: 10.1016/0378-7753(94)80053-7

24 Huang, Z.-H.; Song, Y.; Xu, X.-X.; Liu, X.-X. Ordered Polypyrrole Nanowire Arrays Grown on a Carbon Cloth Substrate for a HighPerformance Pseudocapacitor Electrode. ACS Appl. Mater. Interfaces 2015, 7 (45), 25506- 25513, DOI: 10.1021/acsami.5b08830

25 Vernitskaya, T. V.; Efimov, O. N. Polypyrrole: A Conducting Polymer; Its Synthesis, Properties and Applications. Russ. Chem. Rev. 1997, 66 (5), 443-457, DOI: 10.1070/RC1997v066n05ABEH000261 
26 Huang, M.; Li, F.; Dong, F.; Zhang, Y. X.; Zhang, L. L. MnO2-based Nanostructures for High-Performance Supercapacitors. J. Mater. Chem. A 2015, 3, 21380- 21423, DOI: 10.1039/C5TA05523G

$27 \mathrm{Hu}$, C.-C.; Tsou, T.-W. Ideal Capacitive Behavior of Hydrous Manganese Oxide Prepared by Anodic Deposition. Electrochem. Commun. 2002, 4 (2), 105-109, DOI: 10.1016/S1388-2481(01)00285-5

28 Brousse, T.; Toupin, M.; Dugas, R.; Athouël, L.; Crosnier, O.; Bélanger, D. Crystalline MnO2 as Possible Alternatives to Amorphous Compounds in Electrochemical Supercapacitors. J. Electrochem. Soc. 2006, 153, A2171- A2180, DOI: 10.1149/1.2352197

29 Devaraj, S.; Munichandraiah, N. Effect of Crystallographic Structure of $\mathrm{MnO} 2$ on Its Electrochemical Capacitance Properties. J. Phys. Chem. C 2008, 112 (11), 4406- 4417, DOI: 10.1021/jp7108785

30 Pang, S.-C.; Anderson, M. A.; Chapman, T. W. Novel Electrode Materials for Thin-Film Ultracapacitors: Comparison of Electrochemical Properties of Sol-Gel-Derived and Electrodeposited Manganese Dioxide. J. Electrochem. Soc. 2000, 147 (2), 444- 450, DOI:

$10.1149 / 1.1393216$

31 Zhang, K.; Han, X.; Hu, Z.; Zhang, X.; Tao, Z.; Chen, J. Nanostructured Mn-based oxides for electrochemical energy storage and conversion. Chem. Soc. Rev. 2015, 44, 699- 728, DOI: 10.1039/C4CS00218K

32 Wang, J.-G.; Kang, F.; Wei, B. Engineering of MnO2-Based Nanocomposites for High-Performance Supercapacitors. Prog. Mater. Sci. 2015, 74, 51- 124, DOI: 10.1016/j.pmatsci.2015.04.003

33 Luo, D.; Wallar, C. J.; Shi, K.; Zhitomirsky, I. Enhanced Capacitive Performance of MnO2- Multiwalled Carbon Nanotube Electrodes, Prepared Using Lauryl Gallate Dispersant. Colloids Surf., A 2016, 509, 504- 511, DOI: 10.1016/j.colsurfa.2016.09.065

34 Higgins, T. M.; McAteer, D.; Coelho, J. C. M.; Sanchez, B. M.; Gholamvand, Z.; Moriarty, G.; McEvoy, N.; Berner, N. C.; Duesberg, G. S.; Nicolosi, V.; Coleman, J. N. Effect of Percolation on the Capacitance of Supercapacitor Electrodes Prepared from Composites of Manganese Dioxide Nanoplatelets and Carbon Nanotubes. ACS Nano 2014, 8, 9567- 9579, DOI: 10.1021/nn5038543

35 Tang, X.; Liu, Z.; Zhang, C.; Yang, Z.; Wang, Z. Synthesis and Capacitive Property of Hierarchical Hollow Manganese Oxide Nanospheres with Large Specific Surface Area. J. Power Sources 2009, 193 (2), 939- 943, DOI: 10.1016/j.jpowsour.2009.04.037

36 Wang, P.; Zhao, Y.-J.; Wen, L.-X.; Chen, J.-F.; Lei, Z.-G. Ultrasound-Microwave-Assisted Synthesis of MnO2 Supercapacitor Electrode Materials. Ind. Eng. Chem. Res. 2014, 53 (52), 20116- 20123, DOI: 10.1021/ie5025485

37 Coleman, J. N.; Lotya, M.; O’Neill, A.; Bergin, S. D.; King, P. J.; Khan, U.; Young, K; Gaucher, A.; De, S.; Smith, R. J. Two-Dimensional Nanosheets Produced by Liquid Exfoliation of Layered Materials. Science 2011, 331, 568- 571, DOI: 10.1126/science.1194975

38 Han, T. H.; Lee, W. J.; Lee, D. H.; Kim, J. E.; Choi, E. Y.; Kim, S. O. Peptide/Graphene Hybrid Assembly into Core/Shell Nanowires. Adv. Mater. 2010, 22, 2060- 2064, DOI: 10.1002/adma.200903221

39 Luckham, P. F.; Rossi, S. The Colloidal and Rheological Properties of Bentonite Suspensions. Adv. Colloid Interface Sci. 1999, 82 (1-3), 4392, DOI: 10.1016/S0001-8686(99)00005-6

40 Tanaka, T.; Ebina, Y.; Takada, K.; Kurashima, K.; Sasaki, T. Oversized Titania Nanosheet Crystallites Derived from Flux-Grown Layered Titanate Single Crystals. Chem. Mater. 2003, 15 (18), 3564- 3568, DOI: 10.1021/cm034307j

41 Eda, G.; Yamaguchi, H.; Voiry, D.; Fujita, T.; Chen, M.; Chhowalla, M. Photoluminescence from Chemically Exfoliated MoS2. Nano Lett. 2011, 11, 5111- 5116, DOI: 10.1021/nl201874w

42 Lotya, M.; Hernandez, Y.; King, P. J.; Smith, R. J.; Nicolosi, V.; Karlsson, L. S.; Blighe, F. M.; De, S.; Wang, Z.; McGovern, I. T.; Duesberg, G. S.; Coleman, J. N. Liquid Phase Production of Graphene by Exfoliation of Graphite in Surfactant/Water Solutions. J. Am. Chem. Soc. 2009, 131, 3611-3620, DOI: 10.1021/ja807449u

43 Liu, Z.; Ooi, K.; Kanoh, H.; Tang, W.; Tomida, T. Swelling and Delamination Behaviors of Birnessite-Type Manganese Oxide by Intercalation of Tetraalkylammonium lons. Langmuir 2000, 16 (9), 4154- 4164, DOI: 10.1021/la9913755

44 Omomo, Y.; Sasaki, T.; Wang; Watanabe, M. Redoxable Nanosheet Crystallites of MnO2 Derived via Delamination of a Layered Manganese Oxide. J. Am. Chem. Soc. 2003, 125 (12), 3568- 3575, DOI: 10.1021/ja021364p

$45 \mathrm{Ma}$, R.; Sasaki, T. Two-Dimensional Oxide and Hydroxide Nanosheets: Controllable High-Quality Exfoliation, Molecular Assembly, and Exploration of Functionality. Acc. Chem. Res. 2015, 48 (1), 136- 143, DOI: 10.1021/ar500311w

46 Liu, J.; Essner, J.; Li, J. Hybrid Supercapacitor Based on Coaxially Coated Manganese Oxide on Vertically Aligned Carbon Nanofiber Arrays. Chem. Mater. 2010, 22 (17), 5022- 5030, DOI: 10.1021/cm101591p

47 Huang, M.; Mi, R.; Liu, H.; Li, F.; Zhao, X. L.; Zhang, W.; He, S. X.; Zhang, Y. X. Layered Manganese Oxides-Decorated and Nickel FoamSupported Carbon Nanotubes as Advanced Binder-Free Supercapacitor Electrodes. J. Power Sources 2014, 269, 760- 767, DOI: 10.1016/j.jpowsour.2014.07.031

48 Kim, M.; Hwang, Y.; Kim, J. Super-Capacitive Performance Depending on Different Crystal Structures of MnO2 in Graphene/MnO2 Composites for Supercapacitors. J. Mater. Sci. 2013, 48 (21), 7652- 7663, DOI: 10.1007/s10853-013-7583-3

49 Chen, S.; Zhu, J.; Wu, X.; Han, Q.; Wang, X. Graphene Oxide-MnO2 Nanocomposites for Supercapacitors. ACS Nano 2010, 4 (5), 28222830, DOI: $10.1021 / \mathrm{nn} 901311 \mathrm{t}$

50 Snook, G. A.; Kao, P.; Best, A. S. Conducting-Polymer-Based Supercapacitor Devices and Electrodes. J. Power Sources 2011,196 (1), 112, DOI: 10.1016/j.jpowsour.2010.06.084

51 Duay, J.; Gillette, E.; Liu, R.; Lee, S. B. Highly Flexible Pseudocapacitor Based on Freestanding Heterogeneous MnO 2/Conductive Polymer Nanowire Arrays. Phys. Chem. Chem. Phys. 2012, 14 (10), 3329- 3337, DOI: 10.1039/c2cp00019a

52 Clark, M. P.; Qu, W.; Ivey, D. G. Nanostructured Manganese Oxide and Manganese Oxide/Polyethylenedioxythiophene Rods Electrodeposited onto Nickel Foam for Supercapacitor Applications. J. Appl. Electrochem. 2017, 47, 39- 49, DOI: 10.1007/s10800-016-10154

$53 \mathrm{Ni}$, W.; Wang, D.; Huang, Z.; Zhao, J.; Cui, G. Fabrication of Nanocomposite Electrode with MnO2 Nanoparticles Distributed in Polyaniline for Electrochemical Capacitors. Mater. Chem. Phys. 2010, 124 (2-3), 1151-1154, DOI: 10.1016/j.matchemphys.2010.08.050 54 Lang, X.; Hirata, A.; Fujita, T.; Chen, M. Nanoporous Metal/Oxide Hybrid Electrodes for Electrochemical Supercapacitors. Nat. Nanotechnol. 2011, 6 (4), 232-236, DOI: 10.1038/nnano.2011.13

55 Li, Y.; Fu, H.; Zhang, Y.; Wang, Z.; Li, X. Kirkendall Effect Induced One-Step Fabrication of Tubular Ag/MnOx Nanocomposites for Supercapacitor Application. J. Phys. Chem. C 2014, 118 (13), 6604-6611, DOI: 10.1021/jp412187n 
56 Chen, J.; Huang, Y.; Li, C.; Chen, X.; Zhang, X. Synthesis of NiO@MnO2 Core/Shell Nanocomposites for Supercapacitor Application. Appl. Surf. Sci. 2016, 360, 534-539, DOI: 10.1016/j.apsusc.2015.10.187

57 Wang, Z.; Wang, F.; Tu, J.; Cao, D.; An, X.; Ye, Y. Nickel Foam Supported Hierarchical Mesoporous MnO2/Ni(OH)2 Nanosheet Networks for High Performance Supercapacitor Electrode. Mater. Lett. 2016, 171, 10-13, DOI: 10.1016/j.matlet.2016.02.050

58 Liu, E.-H.; Li, W.; Li, J.; Meng, X.-Y.; Ding, R.; Tan, S.-T. Preparation and Characterization of Nanostructured NiO/MnO2 Composite Electrode for Electrochemical Supercapacitors. Mater. Res. Bull. 2009, 44, 1122- 1126, DOI: 10.1016/j.materresbull.2008.10.003 59 Butel, M.; Gautier, L.; Delmas, C. Cobalt Oxyhydroxides Obtained by 'Chimie Douce' Reactions: Structure and Electronic Conductivity Properties. Solid State Ionics 1999, 122 (1-4), 271-284, DOI: 10.1016/S0167-2738(99)00076-4

60 Godillot, G.; Guerlou-Demourgues, L.; Taberna, P.-L.; Simon, P.; Delmas, C. Original Conductive Nano-Co3O4 Investigated as Electrode Material for Hybrid Supercapacitors. Electrochem. Solid-State Lett. 2011, 14 (10), A139- A142, DOI: 10.1149/1.3609259

61 Ma, B.; Hou, W.; Han, Y.; Sun, R.; Liu, Z.-H. Exfoliation Reaction of Birnessite-Type Manganese Oxide by a Host-Guest Electrostatic Repulsion in Aqueous Solution. Solid State Sci. 2008, 10 (2), 141-147, DOI: 10.1016/j.solidstatesciences.2007.09.003

62 Liu, Z.; Ma, R.; Ebina, Y.; Takada, K.; Sasaki, T. Synthesis and Delamination of Layered Manganese Oxide Nanobelts. Chem. Mater. 2007, 19 (26), 6504-6512, DOI: 10.1021/cm7019203

63 Kim, T. W.; Oh, E.-J.; Jee, A.-Y.; Lim, S. T.; Park, D. H.; Lee, M.; Hyun, S.-H.; Choy, J.-H.; Hwang, S.-J. Soft-Chemical Exfoliation Route to Layered Cobalt Oxide Monolayers and Its Application for Film Deposition and Nanoparticle Synthesis. Chem. - Eur. J. 2009, 15, 1075210761, DOI: 10.1002/chem.200901590

64 Compton, O. C.; Abouimrane, A.; An, Z.; Palmeri, M. J.; Brinson, L. C.; Amine, K.; Nguyen, S. T. Exfoliation and Reassembly of Cobalt Oxide Nanosheets into a Reversible Lithium-Ion Battery Cathode. Small 2012, 8, 1110- 1116, DOI: 10.1002/smll.201101131

65 Kim, J.-Y.; Kim, J.-I.; Choi, S.-M.; Lim, Y. S.; Seo, W.-S.; Hwang, H. J. Nanostructured Thermoelectric Cobalt Oxide by

Exfoliation/Restacking Route. J. Appl. Phys. 2012, 112, 113705, DOI: 10.1063/1.4768258

66 Tang, C.; Giaume, D.; Guerlou-Demourgues, L.; Lefèvre, G.; Barboux, P. Prediction of Isoelectric Point of Manganese and Cobalt Lamellar Oxides: Application to Controlled Synthesis of Mixed Oxides. Langmuir 2018, 34 (23), 6670-6677, DOI: 10.1021/acs.langmuir.8b00190

67 Badot, J.-C.; Ligneel, É.; Dubrunfaut, O.; Guyomard, D.; Lestriez, B. A Multiscale Description of the Electronic Transport within the Hierarchical Architecture of a Composite Electrode for Lithium Batteries. Adv. Funct. Mater. 2009, 19 (17), 2749- 2758, DOI: 10.1002/adfm.200900379

68 McKenna, K. P.; Shluger, A. L. Electron and Hole Trapping in Polycrystalline Metal Oxide Materials. Proc. R. Soc. London, Ser. A 2011, 467, 2043, DOI: $10.1098 /$ rspa.2010.0518

69 Favier, F.; Zhou, Y.; Fournier, C.; Dorval-Douville, G.; Shanley, S.; Jones, D.; Mula, B.; Roziere, J.; Brousse, T.; Belanger, D. Synthesis of Birnessite-Type Layered Manganese Oxide and their Composites for Supercapacitors. ECS Trans 2005, 1, 9- 17, DOI: 10.1149/1.2214605 70 Jagadale, A. D.; Dubal, D. P.; Lokhande, C. D. Electrochemical Behavior of Potentiodynamically Deposited Cobalt Oxyhydroxide (CoOOH) Thin Films for Supercapacitor Application. Mater. Res. Bull. 2012, 47 (3), 672- 676, DOI: 10.1016/j.materresbull.2011.12.029 